\title{
Near-field 3D imaging approach combining MJSR and FGG-NUFFT
}

\author{
WANG Shuzhen ${ }^{1}$, FANG Yang ${ }^{2}$, ZHANG Jin'gang ${ }^{3,4, *}$, LUO Mingshi ${ }^{5}$, and LI Qing ${ }^{6}$ \\ 1. School of Computer Science and Technology, Xidian University, Xi' an 710071, China; \\ 2. School of Electronics and Information, Northwestern Polytechnical University, Xi' an 710072, China; \\ 3. Institute of Optics and Electronics, Chinese Academy of Sciences, Chengdu 610209, China; \\ 4. College of Materials Sciences and Opto-Electronic Technology, University of Chinese Academy of Sciences, Beijing 100049, China; \\ 5. College of Computer Science, Xi' an Shiyou University, Xi'an 710065, China; \\ 6. The Fourth Research Institute of Telecommunications Technology Co.,Ltd, Xi' an 710061, China
}

\begin{abstract}
A near-field three-dimensional (3D) imaging method combining multichannel joint sparse recovery (MJSR) and fast Gaussian gridding nonuniform fast Fourier transform (FGGNUFFT) is proposed, based on a perfect combination of the compressed sensing (CS) theory and the matched filtering (MF) technique. The approach has the advantages of high precision and high efficiency: multichannel joint sparse constraint is adopted to improve the problem that the images recovered by the single channel imaging algorithms do not necessarily share the same positions of the scattering centers; the CS dictionary is constructed by combining MF and FGG-NUFFT, so as to improve the imaging efficiency and memory requirement. Firstly, a near-field 3D imaging model of joint sparse recovery is constructed by combining the MF-based imaging method. Secondly, FGG-NUFFT and reverse FGG-NUFFT are used to replace the interpolation and Fourier transform in MF-based imaging methods, and a sensing matrix with high precision and high efficiency is constructed according to the traditional imaging process. Thirdly, a fast imaging recovery is performed by using the improved separable surrogate functionals (SSF) optimization algorithm, only with matrix and vector multiplication. Finally, a 3D imagery of the near-field target is obtained by using both the horizontal and the pitching interferometric phase information. This paper contains two imaging models, the only difference is the sub-aperture method used in inverse synthetic aperture radar (ISAR) imaging. Compared to traditional CS-based imaging methods, the proposed method includes both forward transform and inverse transform in each iteration, which improves the quality of reconstruction. The experimental results show that, the proposed method improves the imaging accuracy by about $\boldsymbol{O}(\mathbf{1 0})$, accelerates the imaging speed by five times and reduces the memory usage by about $O\left(1^{2}\right)$.
\end{abstract}

\footnotetext{
Manuscript received August 22, 2018.

*Corresponding author.

This work was supported by the National Natural Science Foundation of China (61771369; 61775219; 61640422), the Fundamental Research Funds for the Central Universities (JB180310), the Equipment Research Program of the Chinese Academy of Sciences (YJKYYQ20180039), the Shaanxi Provincial Key R\&D Program (2018SF-409; 2018ZDXM-SF027), and the Natural Science Basic Research Plan.
}

Keywords: interference imaging, joint sparse recovery, compressed sensing (CS), matching filtering (MF), fast Gaussian gridding, nonuniform fast Fourier transform (NUFFT), near-field $3 \mathrm{D}$ imaging.

DOI: $10.21629 / J S E E .2019 .06 .06$

\section{Introduction}

Near-field microwave imaging systems have been successfully developed for applications in the fields of security check, concealed weapon detection, nondestructive testing (NDT), through-wall and inner wall imaging, etc [1-6]. These systems employ microwave probes to scan targets, and two-dimensional/three-dimensional (2D/3D) images are reconstructed from the measurements via synthetic aperture radar (SAR) transform or inverse SAR (ISAR) transform. The near-field microwave imaging system has attracted more and more attention because of the high resolution and wide applications. So far, a large number of near-field imaging algorithms have emerged, such as range migration algorithm (or $\omega-k$ imaging algorithm) [7], polar format imaging [8], back-projection algorithm [9], space-time beamforming imaging [10], nonuniform FFT (NUFFT)-based imaging algorithm [11], and compressed sensing (CS)-based imaging [12].

Since the interference imaging system is easy to implement, this paper focuses on the interference near-field imaging. For the interferometric synthetic aperture radar (InSAR) system [13,14], a 3D image of a target is obtained by proposing the interference phase information of multiantenna. The key to the interferometric imaging techno$\operatorname{logy}$ is the acquisition of high resolution 2D images. Traditional matched filtering (MF)-based near-field imaging methods have some limitations. (i) The images are affected 
by the side lobes, which is not conducive to the extraction of real scatter point information. (ii) In order to achieve high azimuth resolution, a large azimuthal synthetic aperture (or azimuth accumulation angle) is required, which increases the complexity of the antenna motion. (iii) In order to get a higher range resolution, it is necessary to transmit a large bandwidth signal, which greatly increases the data volume.

Recently, the CS theory has been widely used to reduce the amount of data, reduce the sampling rate, and reconstruct with a high probability when the data is undersampled $[15,16]$. The CS theory has several advantages in microwave imaging: enhanced imaging performance, simplified hardware design, and reduced data acquisition time. Since Baraniuk [17] applied CS to microwave imaging for the first time in 2007, the application of CS in microwave imaging has rapidly developed and become an important branch of high-resolution microwave imaging research. In [18], a 2D fast iterative shrinkage-thresholding algorithm (FISTA)-based CS near-field imaging approach was proposed. The method firstly compensates the spherical wave as a plane wave, and then separates the $2 \mathrm{D}$ data into two 1D data for imaging processing. In [19], two methods for the wide-angle 3D image were proposed. A sparsityconstrained regularized least-squares technique is used to form a 3D image in one method. The other one, the TomoSAR approach, is tailored to a particular sparse data collection.

The existing CS-based interferometric 3D imaging has the following problems. (i) Low imaging accuracy. Existing methods do not make full use of the correlation between multiple channels, resulting in errors in interference phase extraction. (ii) Low imaging efficiency. At present, the reconstruction is mainly performed by the echo vectorization into the single measurement vector model, which is very time consuming and takes up a lot of memory.

For the low imaging accuracy of single-channel processing imaging, this paper proposes a 3D imaging algorithm by using multichannel joint sparse recovery (MCJSR). The proposed method, which utilizes the correlation between multi-channel echo signals, improves the reconstruction accuracy, and effectively maintains the relative phase information between channels. A higher-precision interference phase information is obtained, and high-quality $3 \mathrm{D}$ imaging is realized.

The essence of the CS theory is to convert the pressure at the sampling to the signal processing, and its computational complexity is greatly increased. It is mainly reflected in the nonlinear massive operation of recovering from the multi-dimensional information of the sparsely signal. How to achieve fast imaging is the first problem that must be solved. For the rapid reconstruction of the imaging scene, the current research ideas mainly have three directions. (i) Reduce the dimensions of the echo to be processed. In order to avoid the high complexity and large storage capacity brought by the vectorization operation, in [20] a separable perceptual operator framework was designed, which used two low-dimensional measurement matrices to obtain an equivalent vector matrix. In addition, the idea of segmented reconstruction was proposed in [21], which was to reconstruct a large scene after dividing it into many small scenes. However, this method will lose performance. (ii) Reduce the complexity of the algorithm. It mainly includes two aspects, one is to reduce the number of iterations during the reconstruction [22], and the other is to reduce the complexity of each iteration. For example, in [23], the matrix vector multiplication complexity was reduced by using the non-uniform FFT idea. (iii) Combined with the traditional imaging method, the traditional MF idea is used to improve the efficiency. For example, in [24], the MF was combined with CS to realize the efficient reconstruction of SAR images. In order to speed up the reconstruction, this paper takes the following two steps: firstly, combine fast Gaussian gridding-nonuniform FFT (FGG-NUFFT) and $\mathrm{MF}$, construct the dictionary matrix according to the forward SAR/ISAR transform and the R-SAR/R-ISAR transform, and reduce the complexity of the algorithm itself; secondly, adopt the improved SSF algorithm to further accelerate the reconstruction.

We combine MCJSR and FGG-NUFFT, and propose a near-field imaging method, which is based on the advantages of CS, interferometric imaging, and FGGNUFFT. The proposed method combines the CS theory with the MF-based imaging method, and the sensing matrix is constructed according to the traditional MF-based SAR/ISAR imaging process, with which the dictionary scale is reduced. The forward FGG-NUFFT and inverse FGG-NUFFT are correspondingly used in both forward SAR/ISAR and reverse SAR/ISAR in each iteration, which both improve the imaging efficiency and imaging accuracy. Numerical simulations and microwave anechoic chamber experiments show that, compared with the traditional CS-based interferometric imaging methods, the proposed method has the following advantages: (i) improve the recovery precision of strong centers, and preserve effectively the relative phase information between different channels; (ii) accelerate the imaging speed and reduce complexity; (iii) reduce memory usage.

\section{Multi-channel near-field 3D imaging model}

The multi-channel near-field imaging model based on FGG-NUFFT for both SAR and ISAR is established in this 
section. It should be noted that in the large-angle ISAR imaging mode, in order to obtain better imaging quality, the entire imaging interval is generally divided into several sub-apertures with overlapping segments for processing, that is, sub-aperture synthesis.

\subsection{Interferometric ISAR imaging model}

Fig. 1 shows the geometric model of the near-field InISAR imaging system. $y^{\prime}$ is the line of sight of radar, $x^{\prime}$ and $x$ are parallel, $z^{\prime}$ and $z$ are parallel. The distance from the origin of the antenna coordinates to the origin of the target coordinates is $R_{0}\left(R_{0}<\frac{4 D^{2}}{\lambda}, D\right.$ is the maximum size of the target, when the distance $R$ between the imaging observation point and the scattering target body is smaller than the target outer diameter size, it becomes near-field measurement/imaging). The target rotates around the origin of the coordinate, and the angular velocity of rotation is $\omega$. The coordinate of any scattering point $P$ on the target is $(r, \theta, z)$. Then, at the moment of $t$, the distance from the transceiver $l(l \in\{A, B\})$ to the target $P$ is

$R_{l}(r, \theta ; t)=\sqrt{\left(R_{0}+r \cos (\theta+\omega t)\right)^{2}+(r \sin (\theta+\omega t))^{2}+\left(R_{0} \tan \alpha_{i}-z\right)}=\sqrt{\left(R_{0}+y\right)^{2}+x^{2}+\left(R_{0} \tan \alpha_{i}-z\right)^{2}}$

where $\alpha_{i}$ represents the pitch angle of the $i$ th antenna, $(x, y, z)$ is the Cartesian coordinate position of the target.

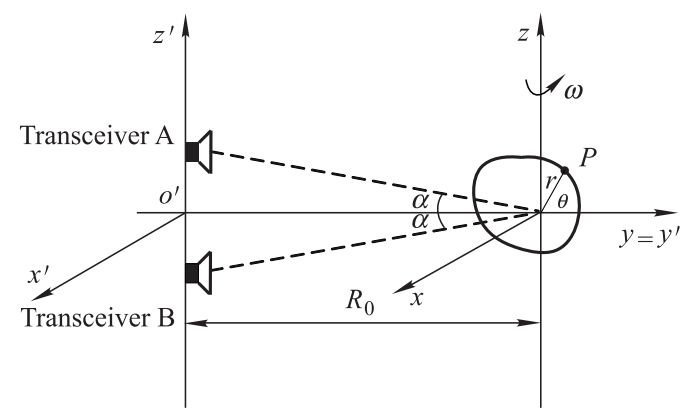

Fig. 1 Geometry model for multi-channel near-field ISAR imaging

The echo signal obtained by antenna A (or B) is

$$
\boldsymbol{S}_{l}(f, \theta)=\iint_{(x, y) \in D} \boldsymbol{g}_{l}(x, y) \exp \left(-\frac{\mathrm{j} 4 \pi f R_{l}}{c}\right) \mathrm{d} x \mathrm{~d} y
$$

where $D$ indicates the area where the imaging scene is located, $\boldsymbol{g}_{l}(x, y)$ represents the scattering coefficient of the target $\boldsymbol{P}(x, y)$.

We express (2) as a summation form:

$$
\boldsymbol{S}_{l}(f, \theta)=\sum_{x=1}^{P} \sum_{y=1}^{Q} \boldsymbol{g}_{l}(x, y) \exp \left[-\mathrm{j} \frac{4 \pi}{\lambda} R_{l}(x, y)\right]
$$

where $P$ and $Q$ represent the number of discretized grids along the $x$ and $y$ axes, respectively.

We discretize the frequency and azimuth samples, where the frequency sampling number is $N$ and the azimuth sampling number is $M$. Then $f_{n}=f_{0}+(n-$ 1) $\Delta f(n=1,2, \ldots, N), \theta_{m}=(m-1) \Delta \theta, m=$ $1,2, \ldots, M$. Equation (3) can be expressed as follows:

$$
\boldsymbol{S}_{l}\left(f_{n}, \theta_{m}\right)=
$$

$$
\sum_{x=1}^{P} \sum_{y=1}^{Q} \boldsymbol{g}_{l}(x, y) \exp \left[-\mathrm{j} \frac{4 \pi f_{n}}{c} R_{l}\left(x, y ; \theta_{m}\right)\right] .
$$

We consider the above ISAR imaging as a wide-angle imaging problem based on the geometric transformation relationships. In wide-angle imaging, the scattering coefficient varies with the angle, namely the anisotropy. We divide the angle equally by using the concept of subaperture for imaging. Then, the echo signal for each $\theta_{m^{\prime}}$ $\left(\theta_{m^{\prime}}=m^{\prime} \theta_{m}, m=1,2, \ldots, M\right)$ angle is expressed as follows:

$$
\boldsymbol{S}_{l-\mathrm{sub}}\left(x^{\prime}, k_{r}\right)=\sum_{x=1}^{P} \sum_{y=1}^{Q} \boldsymbol{g}_{l}(x, y) \exp \left[-\mathrm{j} 2 k_{r} R_{l}\right]
$$

where $x^{\prime}$ is the equivalent moving length of the antenna when the angle is $\theta_{m^{\prime}}, k_{r}=\frac{2 \pi f}{c}\left(f=\sum_{m=0}^{M-1} f_{m}\right)$ refers to the wave number.

\subsection{Interferometric SAR imaging model}

Fig. 2 shows the geometric model of the InSAR near-field imaging system. Transceiver A and transceiver B move along $l_{a}$ and $l_{b}$ respectively, with $l_{a}$ and $l_{b}$ parallel to the $x^{\prime}$ axis. The pitch angles of transceiver A and transceiver $\mathrm{B}$ with respect to the target center are both $\alpha$. The antennas transmit a step frequency signal.

The echo signal obtained by antenna A (or B) is

$$
\boldsymbol{S}_{l}\left(x^{\prime}, k_{r}\right)=\iint_{(x, y) \in \Omega} \boldsymbol{g}_{l}(x, y) \exp \left(-\mathrm{j} 2 k_{r} R_{l}\right) \mathrm{d} x \mathrm{~d} y
$$

where $l \in\{A, B\}, k_{r}=\frac{2 \pi f}{c}\left(f=\sum_{m=0}^{M-1} f_{m}\right)$ refers to the wave number, and $\Omega$ indicates the area where the imaging 
scene is located, $g_{l}(x, y)$ represents the scattering coefficient of the target $P(x, y), R_{l}=\sqrt{\left(x-x^{\prime}\right)^{2}+\left(y+R_{0}\right)^{2}}$ represents the distance from the $l$ th antenna to the target.

The discretized expression of (6) is

$$
\boldsymbol{S}_{l}\left(x^{\prime}, k_{r}\right)=\sum_{x=1}^{P} \sum_{y=1}^{Q} \boldsymbol{g}_{l}(x, y) \exp \left(-\mathrm{j} 2 k_{r} R_{l}\right)
$$

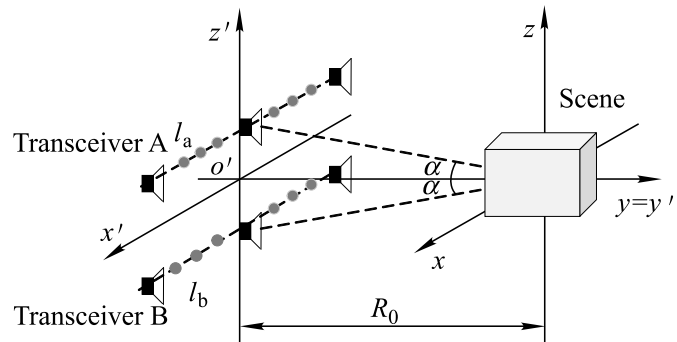

Fig. 2 Geometry model for multi-channel near-field SAR imaging

\subsection{Unified multi-channel near-field imaging model based on FGG-NUFFT}

In the near-field measurement, the target scattered wave is a spherical wave, and the spherical wave is first converted into a superposition of a plurality of plane waves. Solving for $\boldsymbol{g}_{l}(x, y)$ in (4) and (7), we have

$$
\boldsymbol{g}_{l}(x, y)=\boldsymbol{F}_{2 \mathrm{D}}^{-1}\left\{S T\left\{\boldsymbol{F}_{1 \mathrm{D}}\left\{\boldsymbol{S}_{l}\left(x^{\prime}, k_{r}\right)\right\} \mathrm{e}^{-\mathrm{j} \sqrt{4 k_{r}^{2}-k_{x}^{2}} R_{0}}\right\}\right\}
$$

where $k_{x}$ is the Fourier transform variable corresponding to $x^{\prime}, R_{0}$ is the minimum distance between the probes and targets, $\boldsymbol{F}_{2 \mathrm{D}}^{-1}\{\cdot\}$ and $\boldsymbol{F}_{1 \mathrm{D}}\{\cdot\}$ denote the $2 \mathrm{D}$ inverse FFT (IFFT) and the 1D FFT, respectively. $S T\{\cdot\}$ denotes Stolt interpolation.

The frequency is uniformly sampled and the probe scans at the uniform spatial step size. Consequently, $k_{y}$ is non-uniformly distributed and Stolt interpolation is usually used to achieve uniform $k_{y}$ [25]. In (8), the role of $S T\{\cdot\}$ is to correct the range migration in near-field imaging, while regularizing the spectrum to facilitate IFFT. This operation is not only time consuming, but also causes great imaging error. NUFFT is a common method for analyzing non-uniform sampling [26]. In [27], FGGNUFFT was proposed for speeding up NUFFT transformation. In near-field measurements, the frequency samples are non-uniformly sampled, i.e., the samples for $k_{y}$ are non-uniformly sampled, so we perform a 1D NUFFT or NUIFFT for $k_{y}$.

The calculation expression for the 1D NUFFT is

$$
\boldsymbol{F}_{\eta}(k) \approx \sum_{m=0}^{M_{r}-1} \boldsymbol{f}_{\eta}\left(\frac{2 \pi m}{M_{r}}\right) \mathrm{e}^{-\frac{\mathrm{j} k 2 \pi m}{M_{r}}}
$$

where

$$
\begin{gathered}
\boldsymbol{f}_{\eta}\left(\frac{2 \pi m}{M_{r}}\right)=\sum_{i=0}^{N-1} \boldsymbol{f}_{i} \boldsymbol{g}_{\eta}\left(\frac{2 \pi m}{M_{r}}-x_{i}\right)= \\
\sum_{i=0}^{N-1} \boldsymbol{f}_{i} \sum_{l=-\infty}^{\infty} \mathrm{e}^{-\frac{\left(x_{i}-\frac{2 \pi m}{M_{r}}-2 l \pi\right)^{2}}{4 \eta}} \\
\boldsymbol{g}_{\eta}(x)=\sum_{l=-\infty}^{\infty} \mathrm{e}^{-\frac{(x-2 l \pi)^{2}}{4 \eta}} .
\end{gathered}
$$

Details of this algorithm can be found in [27].

Therefore, (8) becomes

$$
\begin{gathered}
\boldsymbol{g}_{l}(x, y)=\boldsymbol{F}_{1 \mathrm{D}, k_{x}}^{-1}\left\{\boldsymbol { F } _ { N U , k _ { y } } ^ { - 1 } \left\{\boldsymbol{F}_{1 \mathrm{D}, x^{\prime}}\right.\right. \\
\left.\left.\left\{\boldsymbol{S}_{l}\left(x^{\prime}, k_{r}\right)\right\} \mathrm{e}^{-\mathrm{j} \sqrt{4 k_{r}^{2}-k_{x}^{2}} R_{0}}\right\}\right\}
\end{gathered}
$$

where $\boldsymbol{F}_{N U, k_{y}}^{-1}\{\cdot\}$ is the one dimensional inverse NUFFT operator in the $k_{y}$ domain, $\boldsymbol{F}_{1 \mathrm{D}, k_{x}}^{-1}\{\cdot\}$ is the one dimensional inverse FFT operator in the $k_{x}$ domain, $\boldsymbol{F}_{1 \mathrm{D}, x^{\prime}}^{-1}\{\cdot\}$ is the one dimensional FFT in the $x^{\prime}$ domain. We can get a $2 \mathrm{D}$ image of the imaging scene in the $l$ th channel through (12).

\section{Accelerating high-precision near-field 3D imaging with MCJSR}

\subsection{Imaging model of joint sparse constrained}

Equation (12) can be described as the matrix form:

$$
\boldsymbol{g}_{l}=\boldsymbol{F}_{1 \mathrm{D}, k_{x}}^{-1}\left\{\boldsymbol{F}_{N U, k_{y}}^{-1}\left\{\boldsymbol{F}_{1 \mathrm{D}, x^{\prime}}\left(\boldsymbol{S}_{l}\right) \cdot \boldsymbol{E}\right\}\right\}=\boldsymbol{A}^{-1} \boldsymbol{S}_{l}
$$

where $\boldsymbol{S}_{l}$ is the observation signal, $\boldsymbol{A}$ is the sensing matrix, and $\boldsymbol{g}_{l}$ is made up of the reflection coefficient of the scene.

Matrix $\boldsymbol{E}$ represents the matched filtering item, and it is defined as follows:

$$
\boldsymbol{E}=\left[\begin{array}{cccccc}
e_{(1,1)} & e_{(1,2)} & \cdots & e_{(1, l)} & \cdots & e_{(1, N)} \\
\vdots & & \ddots & & \ddots & \vdots \\
e_{(M, 1)} & e_{(M, 2)} & \cdots & e_{(M, l)} & \cdots & e_{(M, N)}
\end{array}\right]
$$

The expression of $e_{(i, j)}$ is

$$
e_{(i, j)}=\left[\mathrm{e}^{-\mathrm{j} \sqrt{4 k_{r i}^{2}-k_{x j}^{2}} R_{0}}\right], \quad 1<i<M ; 1<j<N .
$$

Equation (13) represents the forward SAR transform in imaging based on matched filtering, and the reverse SAR transform is formulated as follows:

$$
\boldsymbol{S}_{l}=\boldsymbol{F}_{1 \mathrm{D}, x^{\prime}}^{-1}\left\{\boldsymbol{F}_{N U, k_{y}}\left\{\boldsymbol{F}_{1 \mathrm{D}, k_{x}}\left(\boldsymbol{g}_{l}\right)\right\} \cdot \boldsymbol{E}^{*}\right\}=\boldsymbol{A} \boldsymbol{g}_{l} .
$$


In interference imaging, the echo signals received by the two antennas can be represented as an overall signal:

$$
\boldsymbol{S}=\left[\begin{array}{l}
\boldsymbol{S}_{1} \\
\boldsymbol{S}_{2}
\end{array}\right]=\boldsymbol{A}_{0} \boldsymbol{g}+\boldsymbol{e}=\left[\begin{array}{cc}
\boldsymbol{A} & \mathbf{0} \\
\mathbf{0} & \boldsymbol{A}
\end{array}\right]\left[\begin{array}{l}
\boldsymbol{g}_{1} \\
\boldsymbol{g}_{2}
\end{array}\right]+\left[\begin{array}{l}
\boldsymbol{e}_{1} \\
\boldsymbol{e}_{2}
\end{array}\right]
$$

where $\boldsymbol{S}$ and $\boldsymbol{A}_{0}$ correspond with the overall echo signal and composite dictionary, respectively, $\boldsymbol{g}$ represents the composite scattering coefficient, and $e$ represents composite noise. Since the processing of the echo signals is consistent, the dictionary is also consistent in the interference channel.

When the Nyquist sampling theorem is satisfied, the number of frequency samples is $N$, and the number of azimuth samples is $M$. After sparse sampling (or compressed sampling), the $M^{\prime}\left(M^{\prime} \leqslant M\right)$ and $N^{\prime}\left(N^{\prime} \leqslant N\right)$ group echo data are randomly selected in $M$ and $N$ respectively. The signal model is

$$
\begin{gathered}
\boldsymbol{S}^{\prime}=\boldsymbol{\Phi} \boldsymbol{S}=\left[\begin{array}{cc}
\boldsymbol{\Phi}_{1} & \mathbf{0} \\
\mathbf{0} & \boldsymbol{\Phi}_{2}
\end{array}\right] \boldsymbol{S}=\boldsymbol{\Phi} \boldsymbol{A g}+\boldsymbol{\Phi} \boldsymbol{e}= \\
\boldsymbol{A}^{\prime} \boldsymbol{g}+\boldsymbol{e}^{\prime}
\end{gathered}
$$

where $\boldsymbol{S}^{\prime}$ represents a sparse interference signal with the size of $2 M^{\prime} \times N^{\prime}, \boldsymbol{\Phi}_{i}=\boldsymbol{\Phi}_{i}^{a} \otimes \boldsymbol{\Phi}_{i}^{r}$ represents the sparse sampling matrix of the two channels, $\otimes$ refers to the Kronecker product, $\boldsymbol{A}^{\prime}$ represents a composite sensing matrix.

Solving (18) is an NP-hard problem. When the sensing matrix $\boldsymbol{A}^{\prime}$ satisfies the restricted isometry property (RIP) [28], we can reconstruct the original image with high probability through the optimization technique.

The traditional CS reconstruction method is to vectorized $\boldsymbol{S}$ and $\boldsymbol{g}$. Different from this method, this paper directly processes the $2 \mathrm{D}$ echo signal and imaging. Based on the joint sparse reconstruction model constructed in (18), we will describe in detail the dictionary construction, reconstruction algorithm.

\subsection{Dictionary design combining MF-based imaging technique and FGG-NUFFT}

The combination of the CS theory and the traditional imaging approach will improve the imaging efficiency and accuracy. Based on the description of (13) and (16), we will present a dictionary design process based on the MF-based imaging process and FGG-NUFFT.

According to (13) and (16), the expression of $\boldsymbol{A}$ and $\boldsymbol{A}^{-1}$ can be expressed as matrix multiplication:

$$
\left\{\begin{array}{l}
\boldsymbol{A}=\boldsymbol{F}_{1 \mathrm{D}, x^{\prime}}^{-1} \times \boldsymbol{F}_{N U, k_{y}} \times\left(\boldsymbol{F}_{1 \mathrm{D}, k_{x}} \circ \boldsymbol{E}^{*}\right) \\
\boldsymbol{A}^{-1}=\boldsymbol{F}_{1 \mathrm{D}, k_{x}}^{-1} \times \boldsymbol{F}_{N U, k_{y}}^{-1} \times\left(\boldsymbol{F}_{1 \mathrm{D}, x^{\prime}} \circ \boldsymbol{E}\right)
\end{array}\right.
$$

where $\boldsymbol{A}^{-1}$ and $\boldsymbol{A}$ respectively represent the forward transform and the inverse transform processes of SAR imaging based on matched filtering techniques, $\times$ denotes the matrix product, o denotes the Hadamard product, $\boldsymbol{E}^{*}$ is the complex conjugate of $\boldsymbol{E}$.

$\boldsymbol{F}_{1 \mathrm{D}, k_{x}}$ represents $1 \mathrm{D}$ FFT along $k_{x}$ domain, and is defined as follows:

$$
\boldsymbol{F}_{1 \mathrm{D}, k_{x}}=\frac{1}{\sqrt{M}}
$$

$$
\left[\begin{array}{ccccc}
1 & 1 & 1 & \cdots & 1 \\
1 & \psi & \psi^{2} & \cdots & \psi^{(M-1)} \\
1 & \psi^{2} & \psi^{4} & \cdots & \psi^{2(M-1)} \\
\vdots & \vdots & \vdots & \ddots & \vdots \\
1 & \psi^{(M-1)} & \psi^{2(M-1)} & \cdots & \psi^{(M-1)(M-1)}
\end{array}\right]
$$

with $\psi=\mathrm{e}^{-\frac{2 \pi j}{M}}$.

$\boldsymbol{F}_{N U, k_{y}}$ is the one dimensional NUFFT along $k_{r}$ domain, and is defined as follows:

$$
\begin{gathered}
\boldsymbol{F}_{N U, k_{y}}=\frac{1}{\sqrt{N}} \cdot \\
{\left[\begin{array}{ccccc}
1 & 1 & 1 & \cdots & 1 \\
1 & \omega & \omega^{2} & \cdots & \omega^{(N-1)} \\
1 & \omega^{2} & \omega^{4} & \cdots & \omega^{2(N-1)} \\
\vdots & \vdots & \vdots & \ddots & \vdots \\
1 & \omega^{(N-1)} & \omega^{2(N-1)} & \cdots & \omega^{(N-1)(N-1)}
\end{array}\right]}
\end{gathered}
$$

where $\omega=\mathrm{e}^{-\mathrm{j} k x_{i}}$.

In order to solve $\boldsymbol{g}_{l}$ by (13) with high probability, the dictionary $\boldsymbol{A}$ constructed shall satisfy orthogonality requirements. Since $\boldsymbol{F}_{1 \mathrm{D}, x^{\prime}}, \boldsymbol{F}_{1 \mathrm{D}, k_{x}}^{-1}$ and $\boldsymbol{F}_{N U, k_{y}}$ respectively refer to 1D FFT, 1D IFFT, and 1D NUFFT, then

$$
\left\{\begin{array}{l}
\boldsymbol{F}_{1 \mathrm{D}, x^{\prime}} \boldsymbol{F}_{1 \mathrm{D}, k_{x}}^{-1}=\boldsymbol{I} \\
\boldsymbol{F}_{N U, k_{y}} \boldsymbol{F}_{N U, k_{y}}^{-1}=\boldsymbol{I} .
\end{array}\right.
$$

$\boldsymbol{E} \boldsymbol{E}^{-1}$ refers to a complex diagonal matrix:

$$
\boldsymbol{E} \boldsymbol{E}^{-1}=\operatorname{diag}\left(e_{1}, e_{2}, \ldots, e_{M N}\right)
$$

then

$$
\begin{gathered}
\boldsymbol{A} \boldsymbol{A}^{-1}=\boldsymbol{F}_{1 \mathrm{D}, k_{x}}^{-1} \boldsymbol{F}_{N U, k_{y}} \boldsymbol{E}_{1 \mathrm{D}, x^{\prime}} \\
\left(\boldsymbol{F}_{1 \mathrm{D}, k_{x}}^{-1} \boldsymbol{F}_{N U, k_{y}} \boldsymbol{E} \boldsymbol{F}_{1 \mathrm{D}, x^{\prime}}\right)^{-1}= \\
\boldsymbol{F}_{1 \mathrm{D}, k_{x}}^{-1} \boldsymbol{F}_{N U, k_{y}} \boldsymbol{E}\left(\boldsymbol{F}_{1 \mathrm{D}, x^{\prime}} \boldsymbol{F}_{1 \mathrm{D}, k_{x}}^{-1}\right) \boldsymbol{E}^{-1} . \\
\boldsymbol{F}_{N U, k_{y}}^{-1} \boldsymbol{F}_{1 \mathrm{D}, x^{\prime}}=\operatorname{diag}\left(e_{1}, e_{2}, \ldots, e_{M N}\right) .
\end{gathered}
$$

Thus, dictionary $\boldsymbol{A}$ is orthogonal, and thus $\boldsymbol{\Phi} \boldsymbol{A}$ satisfies the RIP property.

$\boldsymbol{A}^{-1}$ and $\boldsymbol{A}$ represent the forward SAR transform matrix and R-SAR transform matrix, respectively, and their construction processes are shown in Fig. 3. 


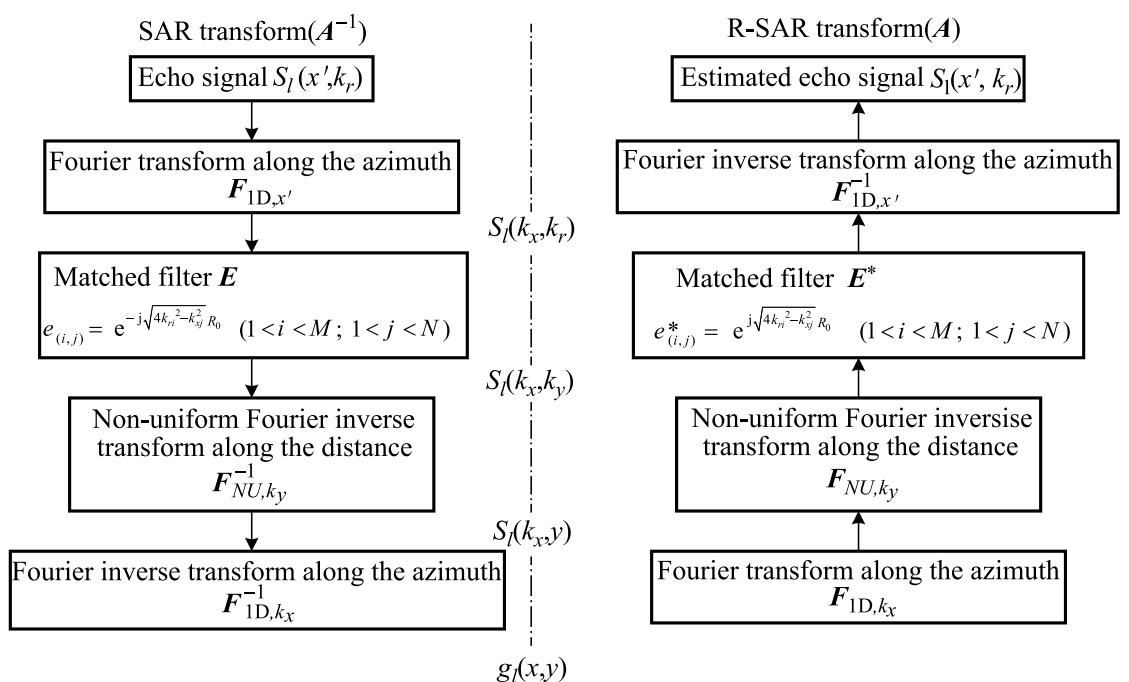

Fig. 3 Imaging flow chart for joint CS and FGG-NUFFT

\subsection{Joint sparse constrained reconstruction algorithm}

In the interferometric imaging system, the observation targets are identical, so the images of each channel have the same target support area, that is, the scattering points on the target are aligned in the respective channel images. The single-channel independent processing imaging method fails to utilize this prior information, so the number and position of scattering points in the reconstructed image of each channel cannot be guaranteed. That is to say, it is impossible to ensure that all scattering points are located in the same pixel of two images. Based on the correlation between multiple channels, we establish a joint sparse solution model:

$$
\|\boldsymbol{g}\|_{2,0}=\left\|\left(\left|\boldsymbol{g}_{1}\right|^{2}+\left|\boldsymbol{g}_{2}\right|^{2}\right)^{\frac{1}{2}}\right\|_{0} .
$$

Based on the joint sparse model constructed in Section 3.1 and (25), we transform near-field interference imaging into joint sparse constraint solving:

$$
\min _{\boldsymbol{g}_{1}, \boldsymbol{g}_{2}}\|\boldsymbol{g}\|_{2,0} \quad \text { s.t. }\left\{\begin{array}{l}
\left\|\boldsymbol{s}_{1}^{\prime}-\boldsymbol{A g}_{1}\right\|_{2}^{2} \leqslant \xi \\
\left\|\boldsymbol{s}_{2}^{\prime}-\boldsymbol{A g}_{2}\right\|_{2}^{2} \leqslant \xi
\end{array} .\right.
$$

According to the Bayesian CS theory, using the maximum posterior probability estimation method, $\boldsymbol{g}_{l}$ can be solved by the following sparse constrained optimization problem [13]:

$$
\begin{gathered}
\min _{\boldsymbol{g}_{1}, \boldsymbol{g}_{2}}\|\boldsymbol{g}\|_{2,0} \\
\text { s.t. }\left\{\begin{array}{c}
\boldsymbol{g}_{1}=\arg \min \left\{\frac{1}{2}\left\|\boldsymbol{S}_{1}^{\prime}-\boldsymbol{A g}_{1}\right\|_{2}^{2}+\lambda\left\|\boldsymbol{g}_{1}\right\|_{1}\right\} \\
\boldsymbol{g}_{2}=\arg \min \left\{\frac{1}{2}\left\|\boldsymbol{S}_{2}^{\prime}-\boldsymbol{A} \boldsymbol{g}_{2}\right\|_{2}^{2}+\lambda\left\|\boldsymbol{g}_{2}\right\|_{1}\right\}
\end{array} .\right.
\end{gathered}
$$

Currently, there are many algorithms to solve the minimization problem of (27), for example, steepest-descent and conjugate-gradient. However, these methods are often found inefficient, requiring too many iterations and too many computations to reach their destination.

Rewrite (27) and express it as a functional form:

$$
f(\boldsymbol{g})=\frac{1}{2}\|\boldsymbol{S}-\boldsymbol{A g}\|_{2}^{2}+\lambda\|\boldsymbol{g}\|_{1} .
$$

We adopt the SSF method based on the proximal point to solve it. Denoting $\boldsymbol{g}_{0}=\boldsymbol{A}^{-1} \boldsymbol{S}$, we get

$$
\begin{gathered}
f(\boldsymbol{g})=\frac{1}{2}\|\boldsymbol{S}-\boldsymbol{A} \boldsymbol{g}\|_{2}^{2}+\lambda\|\boldsymbol{g}\|_{1}= \\
\sum_{k=1}^{m}\left[\frac{1}{2}\left(\boldsymbol{g}_{0}(k)-\boldsymbol{g}(k)\right)^{2}+\lambda|\boldsymbol{g}(k)|\right] .
\end{gathered}
$$

Construct the scalar function from (29), $\eta(\tau)=\lambda|\tau|+$ $0.5\left(\tau_{0}-\tau\right)^{2}$, and its solution is

$$
\tau=\left\{\begin{array}{l}
0, \quad\left|\tau_{0}\right| \leqslant \lambda \\
\tau_{0}-\operatorname{sign}\left(\tau_{0}\right) \lambda, \quad \text { otherwise }
\end{array} .\right.
$$

Add the following to (30):

$$
d\left(\boldsymbol{g}, \boldsymbol{g}_{0}\right)=\frac{c}{2}\left\|\boldsymbol{g}-\boldsymbol{g}_{0}\right\|_{2}^{2}-\frac{1}{2}\left\|\boldsymbol{A g}-\boldsymbol{A} \boldsymbol{g}_{0}\right\|_{2}^{2}
$$

where $c>\left\|\boldsymbol{A}^{-1} \boldsymbol{A}\right\|_{2}=\lambda_{\max }\left(\boldsymbol{A}^{-1} \boldsymbol{A}\right)$, which is used to ensure that the function is a strictly convex function. This new objective function is

$$
\begin{gathered}
\widetilde{f}(\boldsymbol{g})=\frac{1}{2}\|\boldsymbol{S}-\boldsymbol{A} \boldsymbol{g}\|_{2}^{2}+\lambda\|\boldsymbol{g}\|_{1}+ \\
\frac{c}{2}\left\|\boldsymbol{g}-\boldsymbol{g}_{0}\right\|_{2}^{2}-\frac{1}{2}\left\|\boldsymbol{A} \boldsymbol{g}-\boldsymbol{A} \boldsymbol{g}_{0}\right\|_{2}^{2} .
\end{gathered}
$$


Equation (32) is the separable cost function to be used in the proposed algorithm. We expand and recombine (32):

$$
\begin{gathered}
\tilde{f}(\boldsymbol{g})=\frac{1}{2}\|\boldsymbol{S}-\boldsymbol{A} \boldsymbol{g}\|_{2}^{2}+\lambda\|\boldsymbol{g}\|_{1}+\frac{c}{2}\left\|\boldsymbol{g}-\boldsymbol{g}_{0}\right\|_{2}^{2}- \\
\frac{1}{2}\left\|\boldsymbol{A} \boldsymbol{g}-\boldsymbol{A} \boldsymbol{g}_{0}\right\|_{2}^{2}= \\
\frac{1}{2}\|\boldsymbol{g}\|_{2}^{2}+\frac{1}{2}\left\|\boldsymbol{A} \boldsymbol{g}_{0}\right\|_{2}^{2}+\frac{c}{2}\left\|\boldsymbol{g}_{0}\right\|_{2}^{2}-\boldsymbol{S}^{-1} \boldsymbol{A} \boldsymbol{g}+\lambda\|\boldsymbol{g}\|_{1}+ \\
\frac{c}{2}\|\boldsymbol{g}\|_{2}^{2}-c \boldsymbol{g}^{-1} \boldsymbol{g}_{0}+\boldsymbol{g}^{-1} \boldsymbol{A}^{-1} \boldsymbol{A} \boldsymbol{g}_{0}= \\
\text { Const }-\boldsymbol{g}^{-1}\left[\boldsymbol{A}^{-1}\left(\boldsymbol{S}-\boldsymbol{A g}_{0}\right)+c \boldsymbol{g}_{0}\right]+\lambda\|\boldsymbol{g}\|_{1}+\frac{1}{2}\|\boldsymbol{g}\|_{2}^{2} .
\end{gathered}
$$

The constant in the equation are determined by $S$ and $\boldsymbol{g}_{0}$. We define an intermediate expression:

$$
\boldsymbol{V}_{0}=\frac{1}{c} \boldsymbol{A}^{-1}\left(\boldsymbol{S}-\boldsymbol{A g}_{0}\right)+\boldsymbol{g}_{0}
$$

Now we can rewrite $\tilde{f}(\boldsymbol{g})$ as follows:

$$
\begin{gathered}
\tilde{f}(\boldsymbol{g})=\text { Const }-\boldsymbol{g}^{-1} \boldsymbol{V}_{0}+\frac{\lambda}{c}\|\boldsymbol{g}\|_{1}+\frac{1}{2}\|\boldsymbol{g}\|_{2}^{2}= \\
\text { Const }+\frac{\lambda}{c}\|\boldsymbol{g}\|_{1}+\frac{1}{2}\left\|\boldsymbol{g}-\boldsymbol{V}_{0}\right\|_{2}^{2} .
\end{gathered}
$$

This penalty function is exactly the one we minimized for the unitary case, and the solution is given as follows:

$$
\boldsymbol{g}_{\text {out }}=\zeta_{\lambda / c}\left(\boldsymbol{V}_{0}\right)=\zeta_{\lambda / c}\left(\frac{1}{c} \boldsymbol{A}^{-1}\left(\boldsymbol{S}-\boldsymbol{A g}_{0}\right)+\boldsymbol{g}_{0}\right) \text {. }
$$

Equation (36) is a global minimizer of the function $\widetilde{f}(\boldsymbol{g})$ in (32).

So far we manage to convert the original function $f$ to a new function $\widetilde{f}$, for which we are able to get a closedform expression for its global minimizer. Thus, the proposed algorithm is simply an iterative application of (36) in the form:

$$
\boldsymbol{g}_{k+1}=\zeta_{\lambda / c}\left(\frac{1}{c} \boldsymbol{A}^{-1}\left(\boldsymbol{S}-\boldsymbol{A g}_{k}\right)+\boldsymbol{g}_{k}\right) .
$$

Equations (28) to (37) describe the solution process for $\boldsymbol{g}$ in a signal channel. Below we present a joint sparse solution flow for (32).

Task: Find $\boldsymbol{g}$ that meets (26).

Initialization: Initialize $k=0, \boldsymbol{g}_{i, 0}=0, \boldsymbol{r}_{i, 0}=$ $\boldsymbol{S}_{i}-\boldsymbol{A g}_{i, k}=\boldsymbol{S}_{i}$.

Iteration: $k$ adds 1 to each cycle, and apply these steps:

(i) Back-projection: compute

$$
\boldsymbol{e}_{k}=\sum_{i=1}^{2}\left(\left|\left\langle\boldsymbol{r}_{i, k-1}, \boldsymbol{A}^{-1}\right\rangle\right|^{2}\right)^{\frac{1}{2}}
$$

(ii) Shrinkage: compute $\boldsymbol{e}_{i, k}^{*}=\operatorname{Shrink}\left(\boldsymbol{g}_{i, k-1}+\frac{\boldsymbol{e}_{k}}{c}\right)$ with $\lambda$.

(iii) Line search: choose $\mu$ to minimize the real valued function $f\left(\boldsymbol{g}_{i, k-1}+\mu\left(\boldsymbol{e}_{i, k}^{*}-\boldsymbol{g}_{i, k-1}\right)\right)$.

(iv) Update solution: compute $\boldsymbol{g}_{i, k}=\boldsymbol{g}_{i, k-1}+\mu\left(\boldsymbol{e}_{i, k}^{*}-\right.$ $\left.\boldsymbol{g}_{i, k-1}\right)$.

(v) Update residual: compute $\boldsymbol{r}_{i, k}=\boldsymbol{S}_{i}-\boldsymbol{A} \boldsymbol{g}_{i, k}$.

(vi) Stopping rule: if $\left\|\boldsymbol{g}_{i, k}-\boldsymbol{g}_{i, k-1}\right\|_{2}^{2}$ is smaller than some predetermined threshold, stop. Otherwise, apply another iteration.

Output: The result is $\boldsymbol{g}_{i, k}$.

\subsection{Imaging process description}

Fig. 4 shows the detailed and complete flow of the proposed imaging method. The proposed method is suitable for near-field SAR imaging and ISAR imaging. In the imaging process, we equal the near-field ISAR imaging to wide-angle SAR imaging. Therefore, the ISAR imaging is divided into several sub-apertures for imaging processing. In Fig. 4, the dashed box indicates the sub-aperture processing in ISAR imaging.

\section{Experiments and analysis}

In this section, we verify the validity of the proposed method, including the imaging accuracy, imaging efficiency, imaging performance under different sparsities, and imaging performance with different noises. In order to better illustrate the imaging accuracy of the proposed approach, the experimental part adopts a simple target that can better reflect the position information of the scattering point.

\subsection{Near-field InISAR imaging}

Firstly, we validate the algorithm by using generic Backhoe data. Fig. 5 shows the computer aided-design (CAD) model of Backhoe. In the Backhoe data set, two sets of data with pitch angles of $42^{\circ}$ and $42.07^{\circ}$ are selected as the interference imaging raw data, and divide it into 17 sub-apertures. Table 1 shows the detailed parameters.

Table 1 Backhoe electromagnetic simulation parameters

\begin{tabular}{cc}
\hline Parameter & Value \\
\hline Center frequency $/ \mathrm{GHz}$ & 10 \\
Bandwidth/GHz & 6 \\
Sampling number of frequency & 512 \\
Azimuth accumulation angle/ $\left(^{\circ}\right)$ & 51 \\
Sampling number of direction & $71 * 17$ \\
Pitch angle $/\left(^{\circ}\right)$ & 0.07 \\
\hline
\end{tabular}



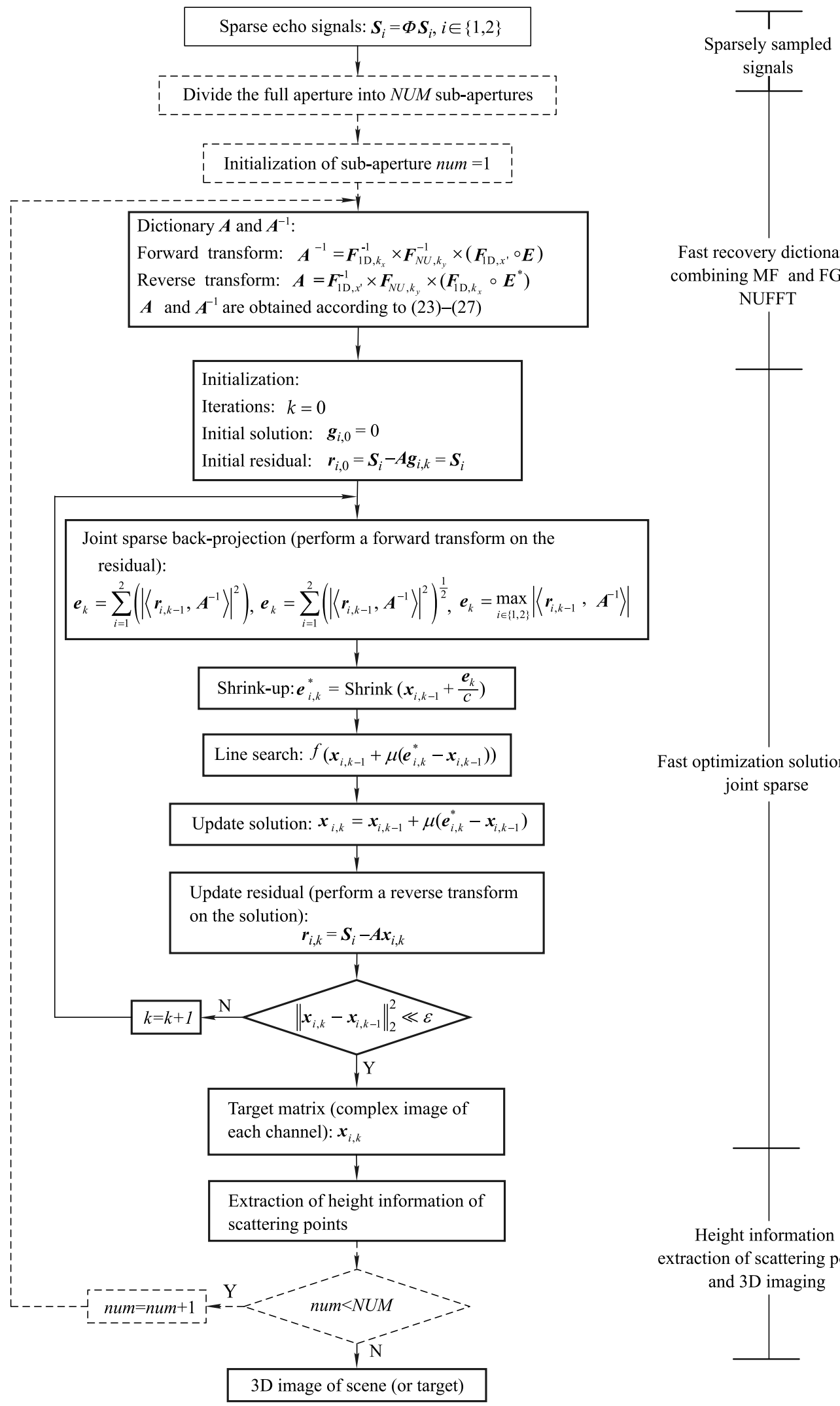

Fast recovery dictionary combining MF and FGG-

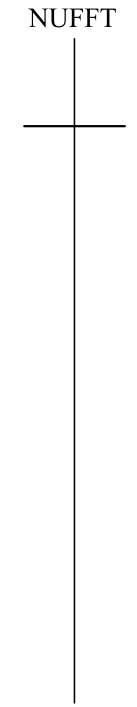

Fast optimization solution for joint sparse

Height information extraction of scattering point and $3 \mathrm{D}$ imaging

Fig. 4 Imaging flow of proposed method 


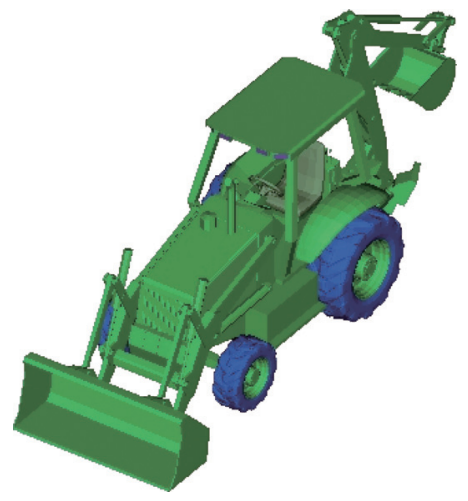

Fig. 5 3D CAD electromagnetic simulation model of Backhoe

Fig. 6 is a comparison of the imaging method proposed with a traditional imaging method. Fig. 6(a), Fig. 6(c), and Fig. 6(e) show the results of imaging by using the singlechannel independently processed CS imaging method proposed in [13]. It can be seen from Fig. 6 that the singlechannel independent processing imaging method has a distorted position of the scattering point and cannot be prepared to describe the target geometric information. The imaging proposed in this paper can accurately reflect the target geometric information. The reason for the above results is that the single-channel independent processing imaging method fails to make full use of the correlation between the channels, resulting in the two images not being in the same imaging pixel unit.

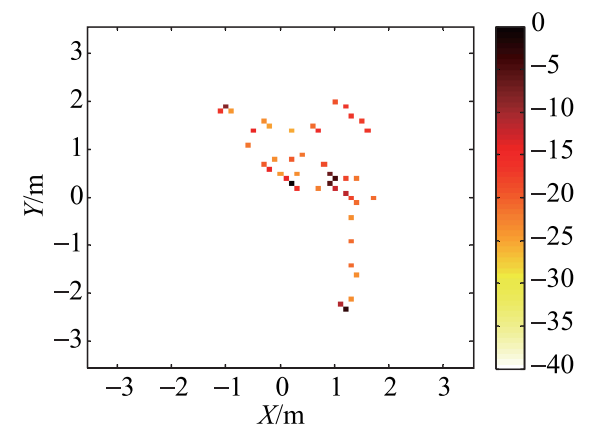

(a) Complex images of channel-1 using traditional imaging processing

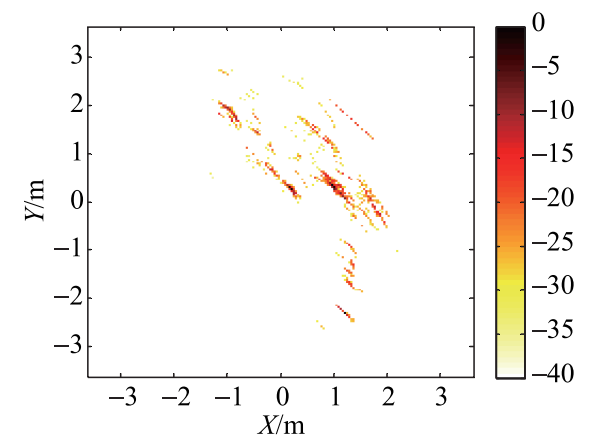

(b) Complex images of channel-1 using imaging processing of the proposed approach

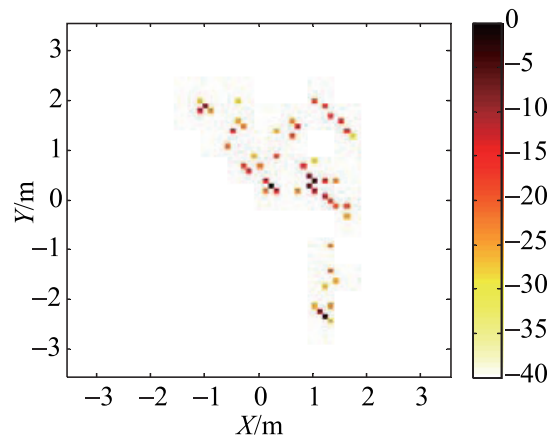

(c) Complex images of channel-2 using traditional imaging processing

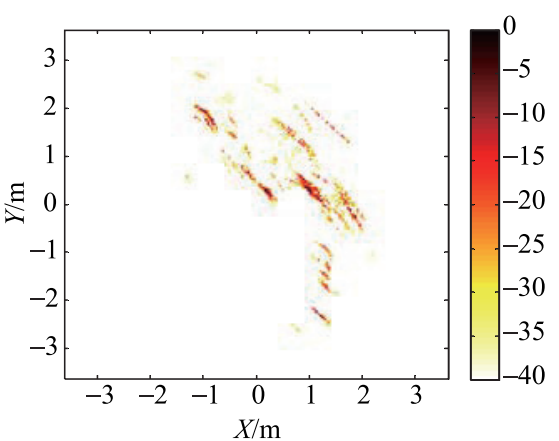

(d) Complex images of channel-2 using imaging processing of the proposed approach

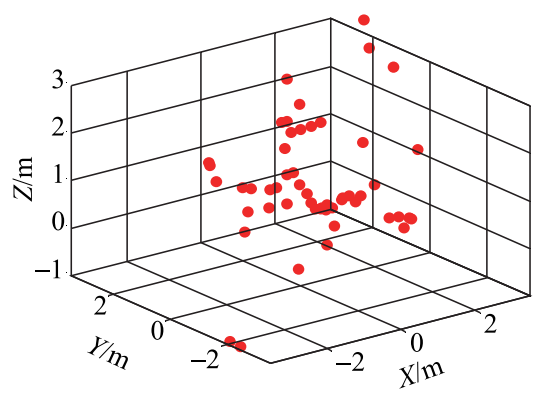

(e) 3D imaging results of the traditional imaging method

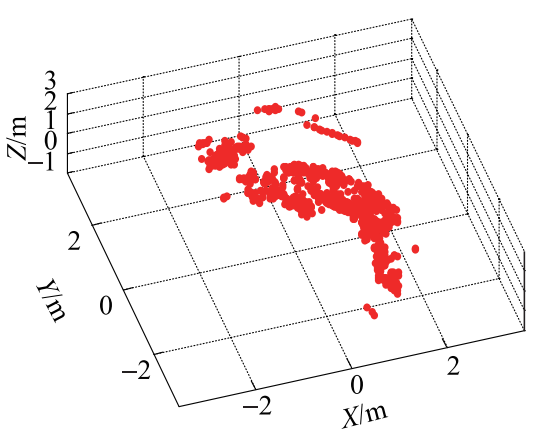

(f) 3D imaging results of imaging processing of the proposed method

Fig. 6 InISAR imaging of Backhoe with complete data

The proposed imaging method achieves the unification of the position of scattering points through multi-channel joint constraint. 


\subsection{Near-field InSAR Imaging}

The imaging system is shown in Fig. 7. Fig. 8 shows the observation target scene, consisting of five metal balls. The synthetic aperture is formed by sliding guide, and the two adjacent scanning paths create two interference trajectories. Imaging parameters are shown in Table 2.

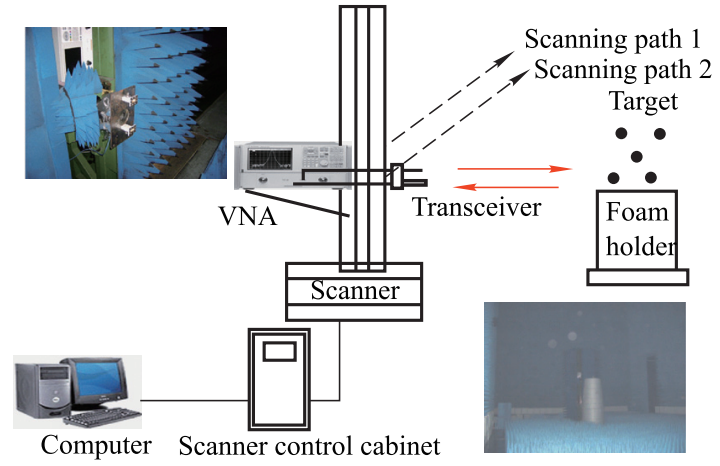

Fig. 7 Near-field InSAR imaging system in anechoic chamber

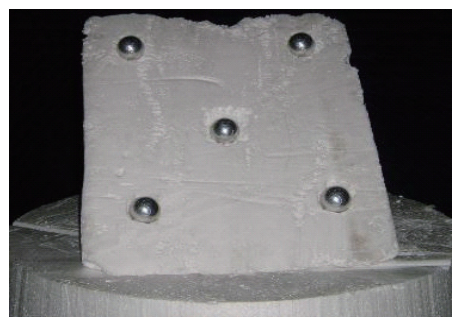

(a) Optical picture of five balls

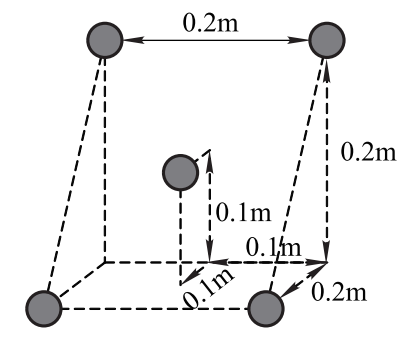

(b) Distribution of target spatial position

Fig. 8 Target position of five metal balls

Table 2 Parameters of InSAR imaging system in anechoic chamber

\begin{tabular}{cc}
\hline Parameter & Value \\
\hline Center frequency $/ \mathrm{GHz}$ & 10 \\
Bandwidth $/ \mathrm{GHz}$ & 4 \\
Interval of frequency sampling $/(\mathrm{MHz} * \mathrm{M})$ & 40 (Sparse sampling) \\
Length of aperture $/ \mathrm{m}$ & 1 \\
Antenna scanning interval $/(\mathrm{m} * \mathrm{~N})$ & 0.02 (Sparse sampling) \\
Baseline length $/ \mathrm{m}$ & 0.02 \\
Distance between antenna and target $/ \mathrm{m}$ & 2 \\
\hline
\end{tabular}

It can be seen from Fig. 9 that the proposed method can accurately reflect the position information of the scattering points.

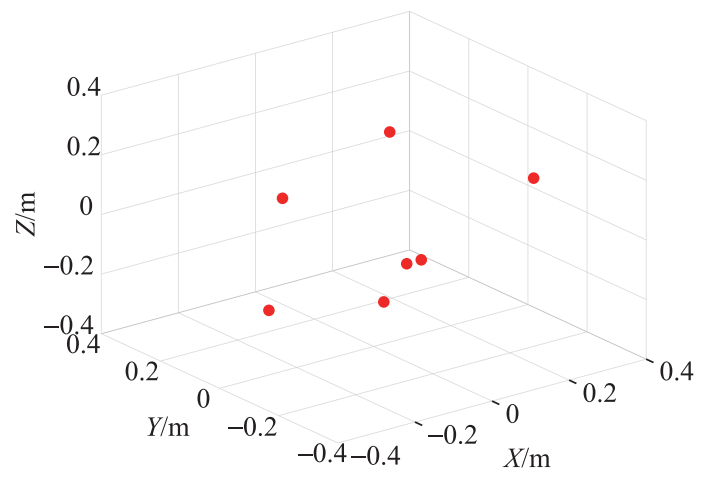

(a) Traditional imaging method

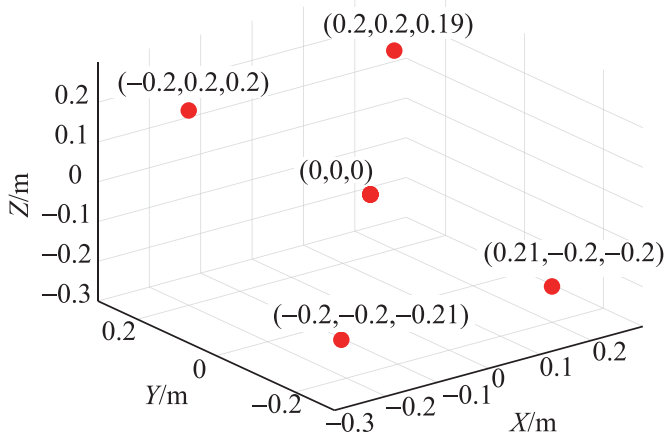

(b) Proposed imaging method

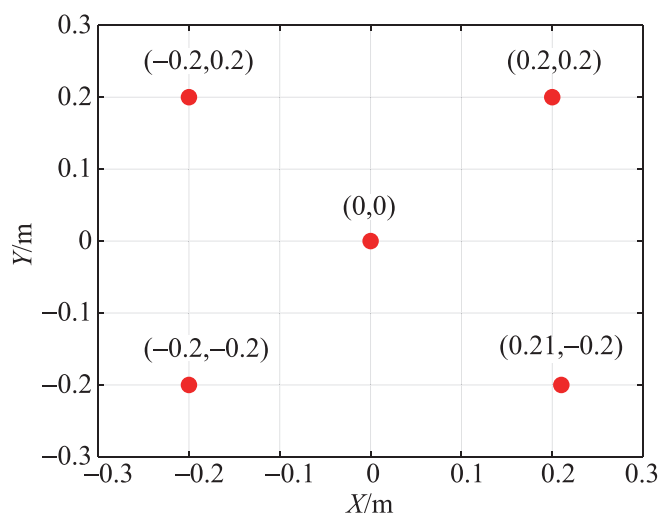

(c) $X$ - $Y$ plane projection

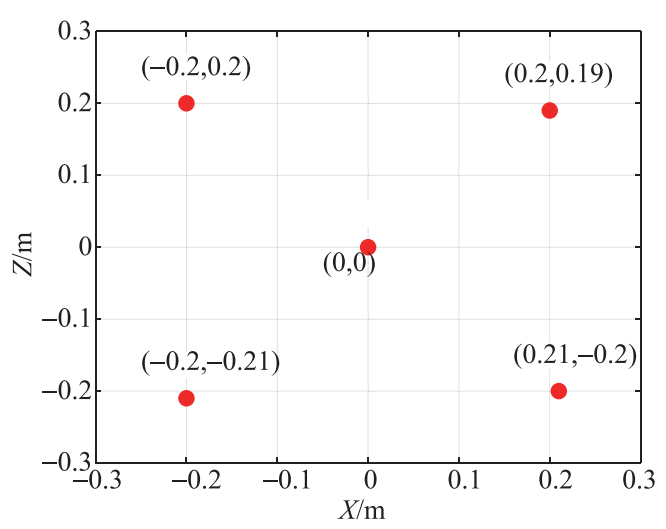

(d) $X$-Z plane projection 


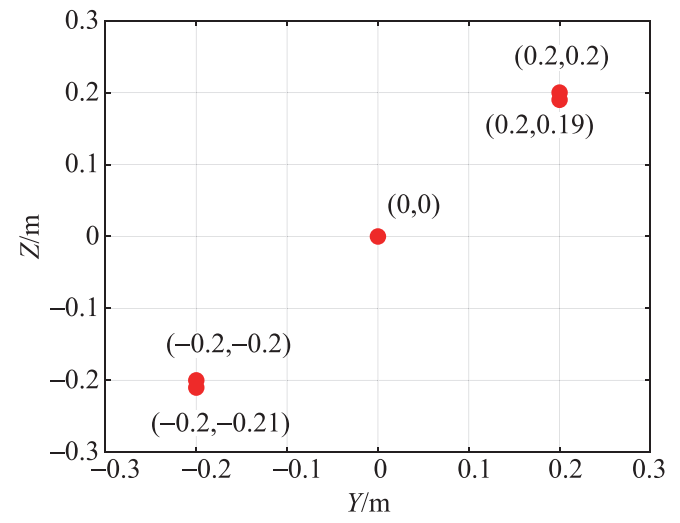

(e) $Y-Z$ plane projection

Fig. 9 Near-field InSAR imaging results of different methods

However, the traditional imaging approach in Fig. 9(a) causes the position of the scattering point to be seriously deviated due to insufficient use of the relevant information between the channels, so that the target scattering point is scattered due to the position mismatch.

\subsection{Performance analysis}

\subsubsection{Imaging accuracy}

We select three typical scattering points from Fig. 6, compare their position information, and quantitatively analyze the imaging accuracy of the proposed method. Table 3 compares the original coordinates of the three scattering points, the coordinates of the conventional imaging method, and the coordinates of the proposed imaging method. It can be seen from Table 3 that the imaging accuracy of the proposed method is significantly better than the conventional imaging method. Compared with the imaging method based on single channel independent processing, the accuracy of the proposed method is improved by $90 \%$. Table 4 is the comparison of scatter point position information extracted by the proposed approach and the traditional approach in Fig. 9.

Table 3 Comparison of positional information of typical scattering points

\begin{tabular}{cccc}
\hline Approach & Target 1 $(x, y, z)$ & Target 2 $(x, y, z)$ & Target 3 $(x, y, z)$ \\
\hline Original coordinate & $(0.0125,0.2688,0)$ & $(0.1438,0.2438,0)$ & $(0.1188,0.1688,0.0467)$ \\
Traditional method & $(0,0.25,0.65)$ & $(0.119,0.229,0.15)$ & $(0.12,0.11,0.15)$ \\
Proposed method & $(0.012,0.266,0)$ & $(0.141,0.241,0.02)$ & $(0.1173,0.16,0.046)$ \\
\hline
\end{tabular}

Table 4 Comparison of scatter point position information extracted by different imaging methods

\begin{tabular}{cccccc}
\hline Approach & Target 1 & Target 2 & Target 3 & Target 4 & Target 5 \\
\hline Theoretical coordinate & $(-0.2,-0.2,-0.2)$ & $(0.2,-0.2,-0.2)$ & $(0,0,0)$ & $(-0.2,0.2,0.2)$ & $(0.2,0.2,0.2)$ \\
Proposed method & $(-0.2,-0.2,-0.21)$ & $(0.21,-0.2 .-0.2)$ & $(0,0,0)$ & $(-0.2,0.2,0.2)$ & $(0.2,0.2,0.19)$ \\
& $(-0.19,-0.2,0.2)$ & $(0.2,-0.18,-0.2)$ & $(0.03,-0.01,0.1)$ & $(-0.2,0.2,0.19)$ & $(0.25,0.19,-0.2)$ \\
Traditional method & $(-0.19,-0.21,-0.2)$ & - & $(0.03,0.01,-0.1)$ & - & $(0.25,0.21,0.2)$ \\
\hline
\end{tabular}

As can be seen from Table 4, the maximum deviation of the target position obtained by the traditional method is 0.4 , the maximum deviation of the target position obtained by the proposed method is 0.01 .

\subsubsection{Interferometric phase}

Fig. 10 and Fig. 11 are the interferometric phase information of the target scattering in Fig. 6 and Fig. 9, respectively.

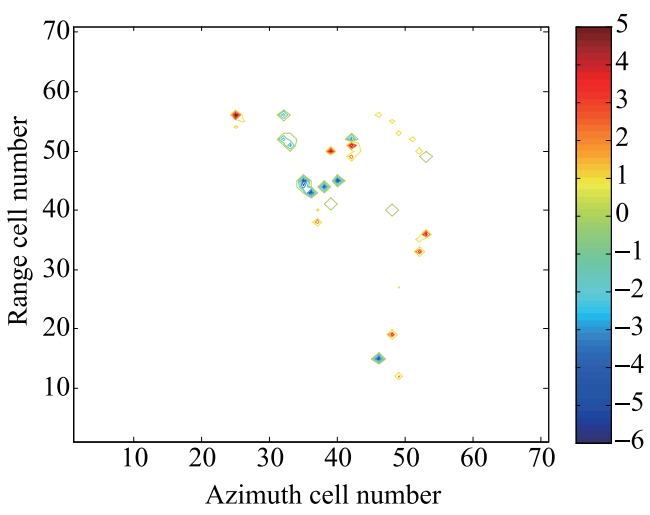

(a) Single channel independent imaging method

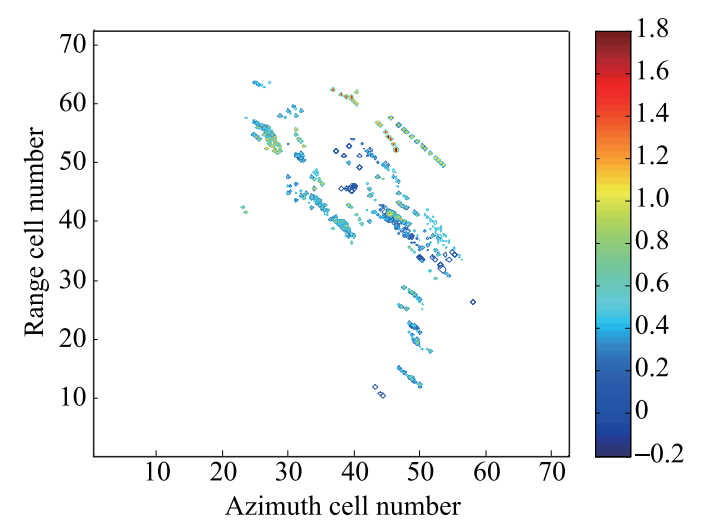

(b) Proposed imaging approach

Fig. 10 Target scattering phase distribution for near-field InISAR 3D imaging

It can be seen in Fig. 10 and Fig. 11 that, for the extraction of the target scattering phase, the performance of the proposed method is superior to the traditional imaging method, and its accuracy and precision are significantly higher. 


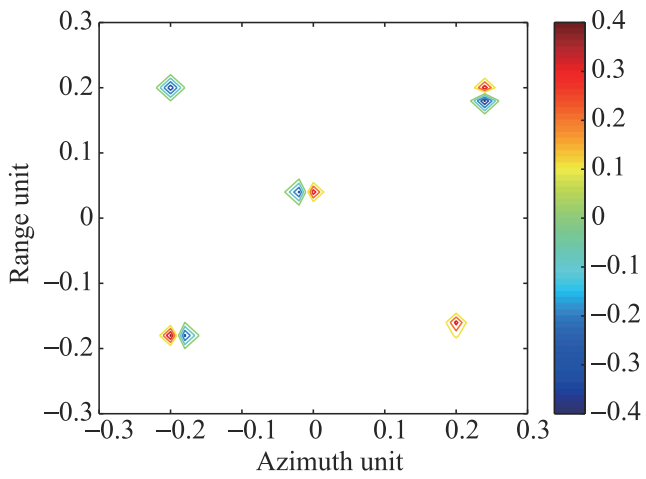

(a) Single channel independent imaging method

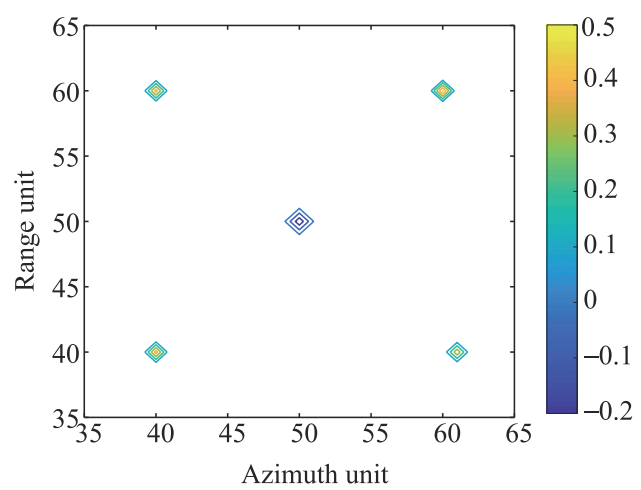

(b) Proposed imaging approach

Fig. 11 Target scattering phase distribution for near-field InSAR 3D imaging

\subsubsection{Anti-noise performance}

In order to verify the performance of the proposed method in the presence of noise, we add $-15 \mathrm{~dB}$ to $20 \mathrm{~dB}$ of Gaussian white noise to the echo signal, and calculate the mean squared error (MSE) estimated on the basis of height. Fig. 12 is a comparison of imaging performance between the proposed imaging method and the traditional imaging method at different SNRs. It can be seen from Fig. 12 that the proposed approach has a better performance to noise suppression than the conventional approach, especially at low SNR, and the average MSE is increased by 0.114 .

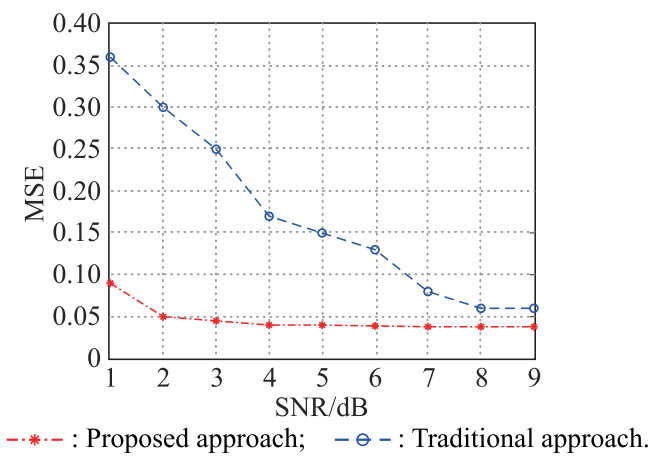

Fig. 12 Comparison for imaging performance of the traditional approach and the proposed approach under different SNRs

\subsubsection{Downsampling imaging performance}

We compare the imaging performance of the proposed method with the traditional method at different sparsity levels. The SNR is fixed as $10 \mathrm{~dB}$. Calculate the MES estimated on the basis of height. It can be seen from Fig. 13 that the proposed approach can still effectively image when it is more than $90 \%$ sparse.

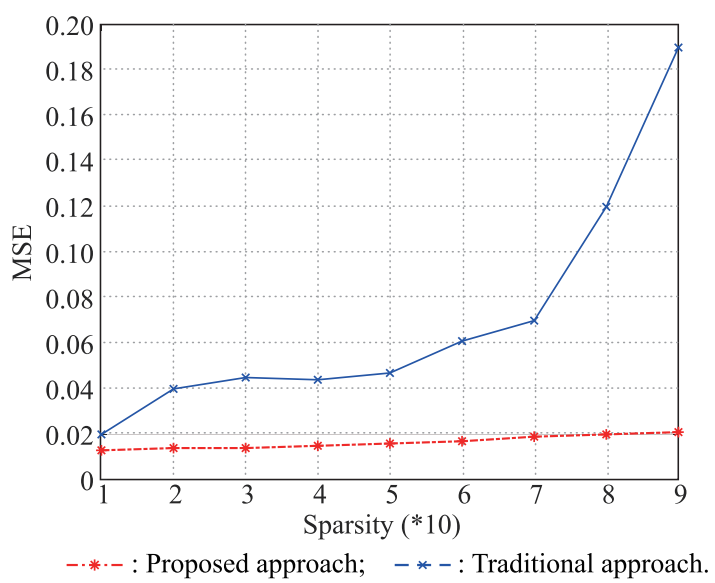

Fig. 13 Imaging performance comparison between the proposed method and the traditional method at different sparse rates

\subsubsection{Imaging computational complexity analysis}

We define the computational complexity as the number of complex multiplications in each imaging iteration. In one-iteration, the computational complexity of the traditional method is $C=2 M N$, and the proposed method is $C^{\prime}=2 * 3 M$, where $M$ represents all the number of samples and $N$ represents all the number of imaging pixels. At the same time, $\boldsymbol{A}$ and $\boldsymbol{A}^{-1}$ can be computed and stored beforehand in the proposed method. The complexity of the traditional imaging method varies with the signal samples and the size of the scene, however, the proposed method is only related to the signal samples. We use the actual calculation time of the computer to show that the proposed method is more efficient. The hardware configuration is CPU of Intel(R) Core(TM) i7-4770HQ CPU @ $2.2 \mathrm{GHz} 2.19 \mathrm{GHz}$, and $8 \mathrm{~GB}$ memory.

Table 5 Comparison of time-consuming of proposed method with the traditional imaging method

\begin{tabular}{l}
\hline Method \\
$\begin{array}{c}\text { Traditional CS imaging method } \\
\text { Proposed method }\end{array}$ \\
\hline The imaging time of the proposed approach is $\frac{1}{6}$ of that \\
of the traditional approach. Taking near-field InSAR imag- \\
ing as an example, we set the number of signal samples \\
to $51 \times 101$. Fig. 14 describes the computational complex- \\
ity of the traditional imaging method and the proposed \\
method.
\end{tabular}




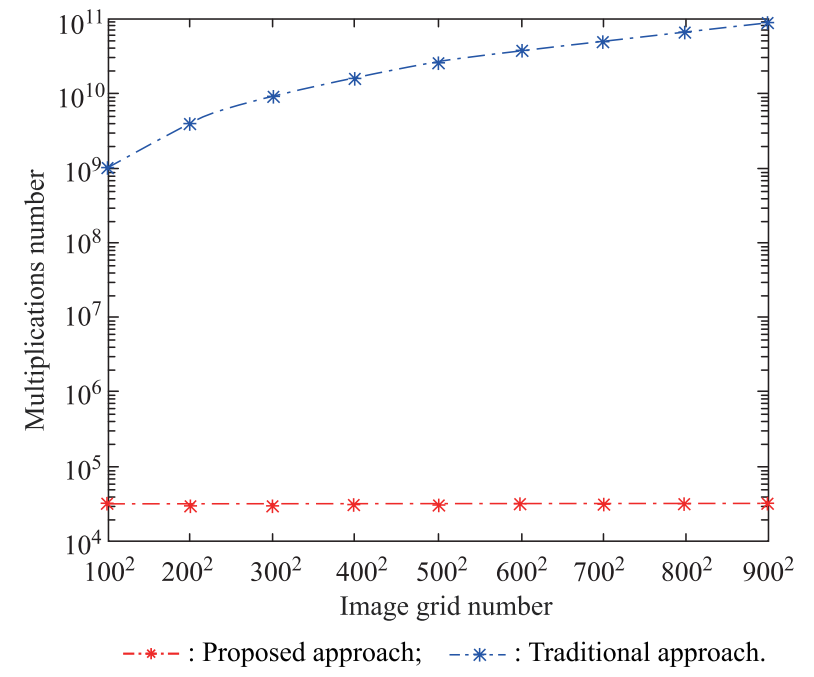

Fig. 14 Comparison of computational complexity of the proposed method with the traditional method

\subsubsection{Analysis of memory requirements}

The size of the sensing matrix in the traditional imaging method is $L \times M \times l \times m$, the size of the sensing matrix in the proposed method is $L \times M$, where $L \times M$ represents the size of signal sampling, $l \times m$ represents the size of the discrete target scene. The memory space required by the traditional imaging method and the proposed imaging method are $2 \times(L \times M) \times(l \times m) \times 4 / 1024 / 10^{6} \mathrm{~GB}$ and $2 \times(L \times M) \times 4 / 1024 / 10^{6} \mathrm{~GB}$.

\section{Conclusions}

Focusing on the near-field interferometric imaging, a 3D imaging approach combining multichannel joint sparse recovery and FGG-NUFFT is presented. Firstly, based on the sparsity of interferometric images, a joint sparse constrained optimal imaging model is established, which effectively utilizes the correlation between multi-channel echo signals, and improves the reconstruction accuracy of scattering points in the images. Secondly, combining the MF technique, SAR transform, R-SAR transform and FGG-NUFFT, a sensing matrix with fast reconstruction performance is constructed. In addition, the improved FSS reconstruction method is used to further accelerate the imaging.

Meeting the near-field interferometric measurement conditions: $R_{0}<\frac{4 D^{2}}{\lambda}$ (near-field); $d \leqslant \frac{\lambda R_{0}}{2 Z_{0}}$ (interferometric condition), where $\lambda$ is the frequency, $R_{0}$ is the distance from the antenna to the target, $Z_{0}$ is the maximum height of the target, and $d$ represents the distance between the two antennas, the imaging method proposed in this paper can effectively work.

This article combines the conventional SAR imaging and sparse theory. The ideas of the constructed dictionary matrix can be widely used in the other mode of SAR imaging. Based on this, we will carry out more experimental experiments, such as security targets, stealth target analysis, etc., while carrying out cylindrical scanning imaging and circular SAR imaging.

\section{References}

[1] FANG Y, WANG B P, SUN C, et al. Near field 3-D imaging approach for joint high-resolution imaging and phase error correction. Journal of Systems Engineering and Electronics, 2017, 28(2): 199-211.

[2] YANG X H, ZHENG Y R, GHASR T, et al. Microwave imaging from sparse measurements for near-field synthetic aperture radar. IEEE Trans. on Instrumentation \& Measurement, 2017, 66(10): 2680-2692.

[3] SHEEN D M, MCMARKIN D L. Three-dimensional radar imaging techniques and systems for near-field applications. Proc. of SPIE, Radar Sensor Technology XX, 2016: 98290V.

[4] ZHANG Y, DENG B, YANG Q, et al. Near-field threedimensional planar millimeter-wave holographic imaging by using frequency scaling algorithm. Sensors, 2017, 17(10): 114.

[5] SHEEN D M, MCMARKIN D L, HALL T E. Near-field threedimensional radar imaging techniques and applications. Applied Optics, 2010, 49(19): 83-93.

[6] LI S Y, ZHU B C, SUN H J. NUFFT-based near-field imaging technique for far-field radar cross section calculation. IEEE Antennas \& Wireless Propagation Letters, 2010, 9(1): 550553.

[7] ZHU R Q, ZHOU J X, JIANG G, et al. Range migration algorithm for near-field MIMO-SAR imaging. IEEE Geoscience and Remote Sensing Letters, 2017, 14(12): 2280-2284.

[8] FORTUNY J. Efficient algorithms for three-dimensional nearfield synthetic aperture radar imaging. Karlsruher, Germany: University of Karslruhe, 2001.

[9] DEMIRCI S, CETINKAYA H, TEKBAS M, et al. Backprojection algorithm for ISAR imaging of near-field concealed objects. Proc. of the URSI General Assembly and Scientific Symposium, 2011: 1-4.

[10] LI X, BOND E J, VAN VEEN B D. An overview of ultra-band microwave imaging via space-time beamforming for earlystage breast-cancer detection. IEEE Antennas and Propagation Magazine, 2005, 47(1): 19-34.

[11] KAN Y Z, ZHU Y F, TANG L, et al. FGG-NUFFT-based method for near-field 3-D imaging using millimeter waves. Sensors, 2016, 16(9): 1-15.

[12] KAJBAF H, CASE J T, YANG Z L, et al. Compressed sensing for SAR-based wideband three-dimensional microwave imaging system using non-uniform fast Fourier transform. IET Radar, Sonar and Navigation, 2013, 7(6): 658-670.

[13] LIU Y B, LI N, WANG R, et al. Achieving high-quality three-dimensional InISAR imaging of maneuvering via superresolution ISAR imaging by exploiting sparseness. IEEE Geoscience and Remote Sensing Letters, 2014, 11(4): 828 - 832.

[14] ZHANG Q, YEO T S, DU G, et al. Estimation of threedimensional motion parameters in interferometric ISAR imaging. IEEE Geoscience and Remote Sensing Letters, 2004, 42(2): $292-300$. 
[15] DONOHO D L. Compressed sensing. IEEE Trans. on Information Theory, 2006, 52(4): $1289-1306$.

[16] CANDES E J, ROMBERG J, TAO T. Robust uncertainty principles: exact signal reconstruction from highly incomplete frequency information. IEEE Trans. on Information Theory, 2006, 52(2): $489-509$.

[17] BARANIUK R, STEEGHS P. Compressive radar imaging. Proc. of the IEEE Radar Conference, 2007: 128-133.

[18] LI S Y, ZHAO G Q, LI H M, et al. Near-field radar imaging via compressive sensing. IEEE Trans. on Antennas and Propagation, 2015, 63(2): 828-833.

[19] AUSTIN C D, ERTIN E, MOSES R L. Sparse signal methods for 3-D radar imaging. IEEE Journal of Selected Topics in Signal Processing, 2011, 5(3): 408-423.

[20] YAIR R, ADRIAN S. Compressed imaging with a separable sensing operator. IEEE Signal Processing Letters, 2009, 16(6): $449-452$.

[21] YANG J G, THOMPSON J, HUANG X T, et al. Segmented reconstruction for compressed sensing SAR imaging. IEEE Trans. on Geoscience Remote Sensing, 2013, 51(7): $4214-$ 4225.

[22] LI S D, CHEN W F, YANG J, et al. A fast complex linearized Bregman iteration algorithm and its application in ISAR imaging. Scientia Sinica Informationis, 2015, 45(9): 1179-1196. (in Chinese)

[23] SUN S L, ZHU G F, JIN T. Novel methods to accelerate CS radar imaging by NUFFT. IEEE Trans. on Geoscience and Remote Sensing, 2015, 53(1): 557-566.

[24] FANG J, XU Z B, ZHANG B C, et al. Fast compressed sensing SAR imaging based on approximated observation. IEEE Journal of Selected Topics in Applied Earth Observations and Remote Sensing, 2014, 7(1): 352-363.

[25] SHEEN D M, MCMARKIN D L, HALL T E. Threedimensional millimeter-wave imaging for concealed weapon detection. IEEE Trans. on Microwave Theory and Techniques, 2001, 49(9): $1581-1592$.

[26] DUTT A, ROKHLIN V. Fast Fourier transforms for nonequispaced data. SIAM Journal on Scientific Computing, 1993, 14(6): $1368-1393$.

[27] LEE G J Y. Accelerating the nonuniform fast Fourier transform. SIAM Review, 2004, 46(3): $443-454$.

[28] CANDÈS E J. The restricted isometry property and its implications for compressed sensing. Comptes Rendus Mathématique, 2008, 364(9-10): 589-592. (in French)

\section{Biographies}
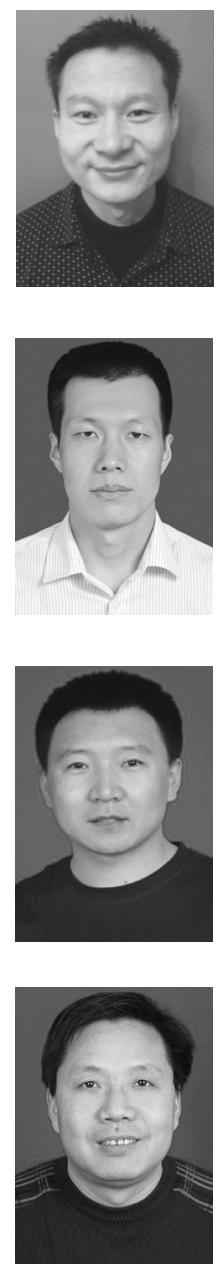

LUO Mingshi was born in 1966. He received his B.S. degree in Beijing University of Posts and Telecommunications in 1988, and M.S. degree in Xi'dian University in 2003. He is now an associate professor at the School of Computer Science, Xi'an Shiyou University. His main research interests include image processing and signal processing.

E-mail: luomsh@xsyu.edu.cn

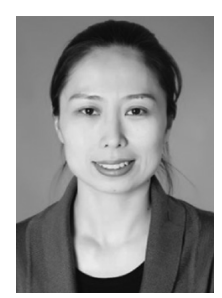

LI Qing was born in 1976. She received her M.S. degree from School of Computer, Xidian University. Currently, she works in the Fourth Institute of Telecommunications Science and Technology Co., Ltd. Her research interest include wireless communication, information security algorithms, radar image, and telemetry antenna.

E-mail: liqing@telfri.net 